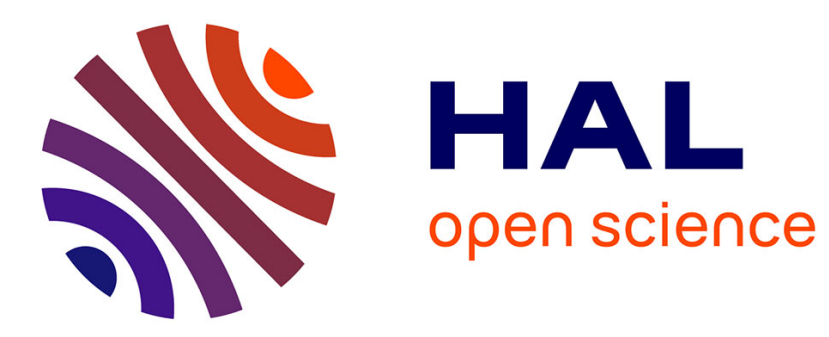

\title{
Le syndrome de Marfan
}

Anne-Marie Vigneron, Frédéric Lioté

\section{To cite this version:}

Anne-Marie Vigneron, Frédéric Lioté. Le syndrome de Marfan. Revue du Rhumatisme monographies, 2019, 86, pp.113 - 119. 10.1016/j.monrhu.2019.02.004 . hal-03486550

\section{HAL Id: hal-03486550 https://hal.science/hal-03486550}

Submitted on 20 Dec 2021

HAL is a multi-disciplinary open access archive for the deposit and dissemination of scientific research documents, whether they are published or not. The documents may come from teaching and research institutions in France or abroad, or from public or private research centers.
L'archive ouverte pluridisciplinaire HAL, est destinée au dépôt et à la diffusion de documents scientifiques de niveau recherche, publiés ou non, émanant des établissements d'enseignement et de recherche français ou étrangers, des laboratoires publics ou privés.

\section{(ㄷ)(1) $\$$}

Distributed under a Creative Commons Attribution - NonCommercial| 4.0 International 
Le syndrome de Marfan

Marfan syndrome

Anne-Marie Vigneron ${ }^{1}$, Frédéric Lioté2, 2,3

${ }^{1}$ Centre de Référence pour le syndrome de Marfan et apparentés, Hôpital Bichat, 75018 Paris,

France

${ }^{2}$ Service de rhumatologie, Pôle appareil locomoteur, centre Viggo Petersen, hôpital Lariboisière, 75010 Paris, France

${ }^{3}$ Inserm UMR1132, centre Viggo Petersen, hôpital Lariboisière, 75010 Paris, France

${ }^{4}$ Université Paris Diderot, USPC, 75010 Paris, France

Auteur correspondant : Frédéric Lioté

Service de rhumatologie, Pôle appareil locomoteur, centre Viggo Petersen, hôpital Lariboisière, 2, rue Ambroise Paré \& 6, rue Guy Patin, 75010 Paris, France

frederic.liote@aphp.fr 


\section{Résumé}

Maladie génétique rare, le syndrome de Marfan est dû le plus souvent à une mutation du gène de la fibrilline de type 1 . Elle affecte principalement les systèmes cardiovasculaire, oculaire et musculosquelettique. Son diagnostic est souvent évoqué dans notre spécialité devant une grande taille, une arachnodactylie, une scoliose, une hyperlaxité ligamentaire selon les critères de Beighton $(>4 / 9)$, des pieds plats, voire une déformation thoracique. Ailleurs la maladie est connue et les complications cardiovasculaires et oculaires sont au premier plan : d'abord un anévrisme de l'aorte et sa complication redoutée, la dissection aortique, mais aussi une ectopie du cristallin.

Le rhumatologue sera appelé pour la prise en charge des douleurs chroniques et/ ou des complications dégénératives (rachialgies sur scoliose dorsolombaire, spondylolisthésis, coxa profunda) chez des malades dont l'espérance de vie a augmenté de façon nette ces dernières années. Les progrès thérapeutiques résident dans l'emploi des $\beta$-bloquants qui ont prolongé la durée de vie des patients en limitant la dilatation de l'aorte et ses complications, et dans le remplacement préventif de l'aorte qui a réduit les dissections. La prise en charge est, au mieux, assurée en centre de référence.

Mots-clés : Syndrome de Marfan ; arachnodactylie ; dolichosténomélie ; scoliose ; Anévrisme aortique ; ectopie du cristallin 


\section{Abstract}

Rare genetic disorder due to fibrillin 1 mutation, Marfan syndrome is resulting in cardiovascular, ocular and skeletal disorders. Rheumatologist might be the first-line physician when facing tall patient with arachnodactily, scoliosis, hypermobility syndrome according to Beigthon's criteria $(>4 / 9)$, flat feet, thoracic deformities. Otherwise the disease has already been diagnosed and cardiovascular involvement including aortic ectasia, or ocular lesions such as ectopia lentis, are of major concern. Rheumatologist can contribute to management of chronic pain or osteoarthritis following scoliosis, spondylolisthesis, or coxopathy. Therapeutic milestones are $\beta$-blockers aiming to reduce the risk of aortic dilation, and prophylactic surgery for preventing aortic dissection. Life span has been dramatically increased over last decades, and management, at least in France, is ideally done in reference centers.

Keywords: Marfan syndrome ; arachnodactily ; dolichostenomely ; scoliosis ; aortic dilatation ; ectopia lentis 


\section{Introduction}

Décrit pour la première fois en 1896, le syndrome de Marfan est une maladie génétique rare qui affecte notamment les systèmes cardiovasculaire, oculaire et musculosquelettique. Sa première description a été rapportée en 1896 par Antonin-Bernard Marfan (1858-1942), pédiatre parisien. Parmi les personnages connus et Marfan présumés, on peut citer Abraham Lincoln, Charles-Maurice Talleyrand, Nicolo Paganini et Serguei Rachmaninov.

Sa prévalence est estimée à un cas sur 5000 naissances en tenant compte des formes incomplètes [1] ; c'est dire que 10.000 à 12.000 personnes sont atteintes de ce syndrome en France. C'est une maladie génétique, de transmission autosomique dominante, la plupart du temps liée à une mutation du gène de la fibrilline de type 1, essentiellement FBN1. Dans un tiers des cas, il s'agit d'une néo mutation (modification d'un gène qui touche de façon isolée et pour la première fois un individu d'une famille). La maladie se caractérise par une hétérogénéité des symptômes cliniques et de l'expression génétique. Elle associe notamment des signes cardiovasculaires, atteinte la plus préoccupante avec dilatation aortique, risque de dissection aortique et de décès [2], une atteinte oculaire et une atteinte musculosquelettique. Sa prise en charge nécessite un avis d'experts (Encadré 1)

\section{Circonstances de découverte}

Dans le cadre de sa pratique quotidienne, le rhumatologue peut être amené à rencontrer des patients atteints d'un syndrome de Marfan dans deux circonstances particulières :

- un morphotype évocateur (grande taille, longs doigts...),

- chez un patient atteint d'un syndrome de Marfan connu, pour le suivi et/ou la prise en charge de l'atteinte musculosquelettique.

\subsection{Morphotype évocateur}

Des signes tels qu'une grande taille, une maigreur, des membres fins et longs, ou encore de grands doigts (arachnodactylie) orientent vers la possibilité d'un syndrome de Marfan, d'autant plus s'ils 
sont associés à une hyperlaxité articulaire, une scoliose, des pieds plats et/ou une déformation du thorax.

\subsection{Anomalies du système musculosquelettique}

Parmi les signes squelettiques, certains sont non spécifiques: scoliose, pieds plats, hyperlaxité articulaire. D'autres orientent plus vers un syndrome de Marfan : arachnodactylie ou doigts dits en toile d’araignée (Fig. 1), pectus carinatum, (Fig. 2), dolichosténomélie.

L'arachnodactylie est mise en évidence par 2 signes, le signe du pouce (le pouce posé dans la paume de la main, poing fermé, dépasse largement le bord cubital) et le signe du poignet (les phalanges distales du pouce et de l'auriculaire enserrant le poignet controlatéral se chevauchent) (Figure 3).

Les déformations thoraciques sont soit vers l'avant (pectus carinatum), soit en creux dit en entonnoir (pectus excavatum).

La dolichosténomélie est définie par une envergure (distance entre l'extrémité du majeur des 2 mains, bras écartés à l'horizontale) trop grande par rapport à la taille avec un rapport Envergure/Taille supérieure à 1,05. L'aspect extérieur des sujets atteints est ainsi caractéristique avec grande taille, grande envergure, maigreur, déformation thoracique, doigts excessivement longs et fins. La taille a été évaluée dans une étude observationnelle de la cohorte de patients suivis dans le centre référent français [3]. Un total de 259 enfants porteurs de la mutation FBN1 et remplissant les critères de diagnostic de Ghent 1 a été comparé à 474 enfants avec mutation négative. À 17 ans, la taille était respectivement de $191 \mathrm{~cm}+$ ou $-8,4 \mathrm{~cm}(+2,9$ DS) dans le groupe avec mutation FBN1 versus $182 \mathrm{~cm}+$ ou $-8,1 \mathrm{~cm}(+1,6$ DS) pour les garçons de l'autre groupe et $178,3 \mathrm{~cm}+$ ou $-7,6 \mathrm{~cm}$ (+2,7 DS) vs $169,5 \mathrm{~cm}+$ ou $-6,8 \mathrm{~cm}$ chez les filles.

II existe un faciès particulier (faciès allongé, micrognathisme, hypoplasie malaire avec pommettes peu saillantes), on peut aussi observer un palais ogival avec chevauchement des dents. L’hyperlaxité articulaire est recherchée par le score de Beighton qui évalue la mobilité des coudes, genoux, pouces et cinquièmes doigts et rachis avec la note de 1 pour une souplesse excessive à 
chaque articulation testée. Un score supérieur à 4/9 définit l'hyperlaxité articulaire. Elle peut coexister avec un flessum des coudes au cours du Marfan, ce qui est insolite.

La protrusion acétabulaire n'a en général, pas de retentissement clinique, elle est découverte sur le cliché de bassin et donne lieu tardivement à une arthrose secondaire. En dehors de la scoliose, l'atteinte du rachis peut se traduire par une cyphose dorsolombaire ou par une déformation des vertèbres lombaires du fait d'une ectasie durale. Celle-ci est le plus souvent asymptomatique, donnant rarement des céphalées. Elle est bien visualisée par l'imagerie (scanner ou IRM).

Les conséquences cliniques de l'atteinte musculosquelettique sont essentiellement fonctionnelles avec des douleurs, parfois esthétiques notamment pour le pectus carinatum ou excavatum. Il s'y associe souvent une fatigue.

Selon les patients et au sein d'une même famille, les manifestations musculo-squelettiques sont variables. Ainsi dans une étude chez 146 patients avec Marfan, rapportée en 2016 par De Maio F et coll [4], l'arachnodactylie était l'atteinte musculosquelettique la plus fréquemment observée chez $60 \%$ des patients. Puis par ordre décroissant les déformations thoraciques étaient présentes pour le pectus carinatum dans $40 \%$ des cas, le pectus excavatum chez $30 \%$ des cas, la dolichosténomélie (37\%), les pieds plats sévères (33\%) avec déformation de l'arrière-pied (21\%). Les déformations du rachis (scoliose ou cyphose dorsolombaire) étaient notées chez $25 \%$ des patients, la protrusion acétabulaire dans $18 \%$ des cas et le flessum des coudes dans $15 \%$ des cas. Dans cette étude, $47 \%$ des patients avaient une ectopie du cristallin et $37 \%$ avaient eu une chirurgie cardiaque dont 11/54 en urgence pour dissection aortique. Aucune corrélation n'était notée entre l'étendue de l'atteinte musculo-squelettique et les complications aortiques.

\subsection{Autres symptômes}

\subsubsection{Atteinte cardiaque}

L'atteinte cardiaque se caractérise par une dilatation aortique prédominant au sinus de Valsalva. La dilatation progresse en moyenne de $0,5 \mathrm{~mm}$ par an ce qui justifie une surveillance annuelle par 
échographie cardiaque [5]. La dissection aortique est le risque majeur; elle était auparavant le signe révélateur et le plus souvent fatal de la maladie. Le prolapsus mitral est fréquemment observé, modéré dans l'ensemble.

\subsubsection{Atteinte oculaire}

Les conséquences du syndrome de Marfan sur le système oculaire se traduisent par une ectopie du cristallin supérieure et temporale, signe le plus fréquent. D'autres signes oculaires peuvent être observés: cornée plate, myopie secondaire à une longueur axiale augmentée. La surveillance ophtalmologique doit aussi être annuelle.

\subsubsection{Atteinte cutanée}

L'atteinte cutanée se traduit par la présence de vergetures, non associées à la grossesse ou à des changements de poids. Certaines localisations sont évocatrices de syndrome de Marfan (épaules et rachis lombaire) (Figure 4) [6]. Une autre manifestation cutanée ou plutôt pariétale, ce sont des hernies récidivantes. II n’y a pas d'hyper-élasticité cutanée.

\subsubsection{Atteinte pulmonaire}

L'atteinte du système respiratoire est possible ; la maladie peut engendrer un pneumothorax, chez 5 à $10 \%$ des patients, ou des bulles emphysémateuses apicales [7]. Un syndrome d'apnée du sommeil est fréquent, pouvant contribuer par lui-même à la dilatation aortique. Certaines études suggèrent un rôle éventuel des anomalies cranio-faciales dans la pathogénie de l'apnée du sommeil chez les Marfan.

\subsection{Suivi rhumatologique d'un syndrome de Marfan connu}

Le patient atteint d'un syndrome de Marfan peut consulter un rhumatologue pour le suivi et/ou la prise en charge de l'atteinte musculosquelettique. Comme le pronostic de l'atteinte cardiaque a été transformé avec le traitement $\beta$-bloquant et la chirurgie cardiaque préventive, il est à l'heure actuelle courant de voir des patients âgés de plus de 40 ans. L'espérance de vie a progressé de 30 ans ces 30 dernières années. 
Les douleurs souvent chroniques peuvent être dorsolombaires en rapport avec la scoliose, le spondylolisthesis ou l'atteinte articulaire postérieure et/ou localisées aux articulations périphériques en lien avec l'arachnodactylie, l'hyperlaxité et/ou l'arthrose.

\section{Formes particulières}

\subsection{Formes apparentées}

D'autres mutations que celle du gène FBN1 ont été décrites (TGFBR1, TGFBR2, SMAD3, ACTA 2, etc.) et se caractérisent par des symptômes particuliers [4], résumés dans le tableau 1.

Ces particularités cliniques doivent être recherchées chez tout(e) patient(e) avec suspicion de syndrome de Marfan car orientant vers une recherche génétique plus spécifique.

La mutation dans le gène SMAD3 [6,8-10] peut interpeller le rhumatologue du fait d'une arthrose précoce avant 40 ans. Les lésions radiologiques sont celles habituellement observées au cours des arthropathies microcristallines telles que pincement scaphotrapézien, calcifications du ligament triangulaire du carpe, avec des localisations particulières ( $2^{\mathrm{e}} \mathrm{MCP}$, tarse). On peut observer une atteinte méniscale, une ostéochondrite disséquante. II s'y associe d'autres manifestations cliniques : neuropathie de type Charcot-Marie-Tooth, asthme, conjonctivite allergique, troubles veineux, atteinte auto-immune (syndrome de Gougerot-Sjögren, thyroïdite de Hashimoto, présence de FAN, d'anticorps anti-ECT, anti-DNA...) [11].

Enfin, l'autre syndrome apparenté au syndrome de Marfan est constitué par le tableau d'anévrysme isolé de l'aorte ascendante mais ces formes-là ne sont pas vues par le rhumatologue mais par le cardiologue [9].

\subsection{Enfant et adolescent}

Les signes squelettiques apparaissent progressivement au cours de l'enfance vers 6-7 ans en moyenne.

La dolichosténomélie (rapport de l'envergure des membres supérieurs sur la taille supérieure à 1,05) est un bon signe de présomption chez l'enfant. Les pieds plats très fréquents jusqu'à l'âge de 4-5 ans 
chez tous les enfants ne sont pas évocateurs dans cette tranche d'âge (Figure 5). Les doigts longs et fins (arachnodactylie) peuvent être à l'origine, chez l'enfant de difficultés en motricité fine (écriture, graphisme) nécessitant une prise en charge adaptée (psychomotricité, ergothérapie) et une adaptation en milieu scolaire : PAI (Plan d'Accueil Individualisé), 1/3 temps, ordinateur en classe ....

La scoliose au cours du Marfan est en général plus précoce et sévère que la scoliose idiopathique. Elle peut s'aggraver au moment de la puberté. Elle nécessite un avis orthopédique avec un suivi clinique et radiologique. La technique EOS au rachis, dix fois moins irradiante que le grand cliché dorsolombaire, prend tout son intérêt chez les enfants/adolescents, où le suivi de la scoliose est rapproché pour adapter au mieux la conduite thérapeutique (surveillance simple, corset, chirurgie...).

Une étude du Centre de Référence pour le syndrome de Marfan et apparentés, en 2014, a montré que la prévalence des signes musculo-squelettiques varie en fonction de l'âge ; ainsi les déformations thoraciques passent de $43 \%$ dans la tranche d'âge $0-6$ ans à $62 \%$ chez les 15-17 ans, l'arachnodactylie augmente de 28 à $67 \%$, la scoliose de 16 à $59 \%$ tandis que l'hypermobilité diminue de 67 à $47 \%$ et les pieds plats de 73 à $65 \%$. La taille paraît un critère simple et discriminant quand elle est supérieure à 3,3 DS. L'ectopie du cristallin et la dilatation aortique, stables au cours du temps (66 à $72 \%$ et 75 à $80 \%$ respectivement), sont également des facteurs discriminants. Enfin, les vergetures augmentent très nettement au fil des ans passant de 2 à $84 \%$ [12].

\subsection{Grossesse}

La grossesse n'est pas contre-indiquée chez la femme porteuse d'un syndrome de Marfan si le diamètre de l'aorte est inférieur à $45 \mathrm{~mm}$. Dans tous les cas, elle doit être discutée avec l'équipe pluridisciplinaire, notamment le cardiologue, et être suivie de façon rapprochée (échographie cardiaque à la fin des $1^{\text {ers }}$ et $2^{\text {es }}$ trimestres de grossesse et tous les mois au cours du $3^{\mathrm{e}}$ trimestre). Le type d'accouchement dépendra du diamètre aortique entre autres. Le $\beta$-bloquant doit être poursuivi tout au long de la grossesse [13-15]. 
Les moyens contraceptifs peuvent être utilisés au cours du Marfan sauf le stérilet en raison du risque d'endocardite infectieuse.

Un diagnostic prénatal peut être proposé chez les couples dont l'un est porteur de la mutation génétique [16]. Une biopsie de trophoblaste est pratiquée entre la $11^{\mathrm{e}}$ et la $13^{\mathrm{e}}$ semaine de grossesse avec une réponse 15 jours plus tard et une consultation pour les résultats avec le (la) généticien(ne) et le (la) psychologue. Si le fœtus est porteur du gène muté, une décision d'interruption volontaire de grossesse (IVG) pourra être prise si le couple confirme la demande initiale d'IVG [17].

\section{Diagnostic}

Le diagnostic ne se pose en fait qu'en cas de formes incomplètes [17]. En cas de doute, un avis cardiologique avec échographie cardiaque et ophtalmologique est demandé afin de progresser dans la démarche diagnostique ou une hospitalisation en hôpital de jour au centre référent est sollicitée.

\subsection{Critères majeurs et mineurs}

Le diagnostic de syndrome de Marfan repose sur un ensemble de critères. Parmi ces critères, certains sont majeurs, d'autres mineurs pour chaque système atteint. II est facilement posé en cas d'antécédent familial avec atteinte d'au moins 2 systèmes et au moins un critère majeur et en l'absence d'histoire familiale lorsque 3 systèmes sont atteints avec au moins 2 critères majeurs.

Le tableau 2 résume l'ensemble des systèmes atteints au cours du Marfan selon la classification de Ghent de 1996 [18]. La liste de critères a été revue en 2010 [19], car au fil des années, les critères cliniques se sont affinés et la génétique a été intégrée au diagnostic.

Dans la nouvelle classification proposée en 2010, certains critères cliniques ont pris plus de poids, comme le pneumothorax de cotation 2, alors que c'était un critère mineur dans la classification précédente [20]. Sur le plan squelettique, l'arachnodactylie est désormais cotée à 3 en cas de signe du poignet et du pouce, et les pieds plats à 1 et à 2 en cas de déformation associée de l'arrière pied. En revanche, le spondylolisthésis, l'hyperlaxité articulaire et le palais ogival ne font plus partie de cette classification [19]. Avec cette nouvelle classification, le diagnostic de Marfan est porté 
dès que la cotation est supérieure ou égale à 7 en tenant compte de la cotation obtenue dans tous les systèmes.

\subsection{Syndromes marfanoïdes}

Dans des formes modérées ou des suspicions diagnostiques, la suspicion justifie de faire une échographie cardiaque à visée diagnostique: recherche de dilatation de l'orifice aortique en particulier ou de confier le patient au Centre Référent.

La demande de test génétique est régulée par le Centre de Référence pour le syndrome de Marfan et apparentés; c'est une recherche longue et coûteuse car le gène est long et les mutations nombreuses, chaque famille atteinte a une mutation qui lui est propre, les résultats sont rendus avec 2 ans de délai en moyenne. Elle n'est donc demandée qu'en cas de forte suspicion diagnostique.

L'hyperlaxité avec flessum du coude, inexpliqué à ce jour, est une association spécifique de la maladie de Marfan, non retrouvée dans la forme hyperlaxe d'Ehlers-Danlos.

Une revue récente de 2017 fait le point entre les différences observées dans le syndrome de Marfan, le syndrome d'Ehlers-Danlos et le syndrome de Loeys-Dietz [21].

\section{Prise en charge thérapeutique}

\subsection{Activités sportives}

Une fois le diagnostic de Marfan établi, il est important de contre-indiquer les sports violents, car ils risquent de favoriser la dilatation aortique en cas d'élévation de pression artérielle lors d'un effort intense et brusque. II existe aussi un risque de luxation du cristallin lors de sports de combat. Les compétitions sont interdites [22].

\subsection{Atteinte cardiaque}

La base du traitement repose d'une part sur le suivi (échographie cardiaque annuelle), d'autre part sur les $\beta$-bloquants. C'est le seul médicament dont l'action sur la dilatation aortique est démontrée [1] et qui a permis de diminuer de façon drastique le risque de dissection aortique qui est très limité si le diamètre aortique est inférieur à $50 \mathrm{~mm}$. L'espoir fondé par l'adjonction 
aux $\beta$-bloquants d'un sartan n'a pas été confirmé par l'étude menée dans le Centre Référent [23]. Chez 303 patients Marfan, d'âge moyen 30 ans, l'évolution du diamètre aortique au sinus de Valsalva n'a pas été modifiée par l'adjonction de losartan. II n'y a pas eu non plus de bénéfice démontré avec les inhibiteurs de l'enzyme de conversion. Le $\beta$-bloquant est recommandé dès la confirmation du diagnostic de Marfan en cas de dilatation aortique et sans dilatation aortique dès l'âge de 4 ans en présence d'une mutation génétique. II doit être poursuivi après intervention cardiaque et au cours de la grossesse. En cas d'intolérance ou de contre-indication aux $\beta$-bloquants (asthme sévère), l'alternative est le vérapamil, inhibiteur calcique (Isoptine ${ }^{\circledR}$ ) [24].

Une intervention cardiaque préventive est décidée quand la dilatation aortique dépasse le seuil de $50 \mathrm{~mm}$ : remplacement de l'aorte initiale et valve biomécanique avec réimplantation des coronaires ou actuellement bioprothèse ne nécessitant pas comme en cas de valve biomécanique, la prise d'anticoagulants, intervention de valve sparing au cours de laquelle la valve est conservée. Lorsque le diamètre aortique est entre 46 et $50 \mathrm{~mm}$, la décision opératoire se fait au cas par cas. La chirurgie peut être proposée en cas d'augmentation de plus de $2 \mathrm{~mm}$ entre deux mesures ou de forme sévère [25].

Si le patient est suivi par le Centre Référent Multidisciplinaire Marfan, une hospitalisation en hôpital de jour est prévue tous les 3 ans pour les adultes et tous les 2 ans chez l'enfant. Lorsque le diagnostic est peu probant chez l'enfant, il est revu de principe à 18 ans pour écarter toute possibilité éventuelle de la maladie. Une carte de soins et d'urgence «Syndrome de Marfan » est remise au patient avec un volet "soins" destiné aux professionnels de santé et un volet « informations et conseils » destiné aux patients et à leur entourage et à porter sur soi en permanence.

\subsection{Atteinte musculosquelettique}

La prise en charge des douleurs chroniques repose sur les antalgiques, les AINS en cas de poussée douloureuse et en l'absence de prise concomitante d'anticoagulants nécessités par une 
valve mécanique. La rééducation du rachis avec renforcement musculaire, exercices d'autoétirement est prescrite en complément ainsi que la rééducation proprioceptive des articulations périphériques en cas d'hyperlaxité avec physiothérapie à visée antalgique, balnéothérapie voire cures thermales.

Les déformations thoraciques ont un retentissement essentiellement esthétique. À noter que si le patient est opéré pour la dilatation aortique, le pectus carinatum est souvent amélioré au décours de la chirurgie cardiaque. Quant au pectus excavatum, il n'est que rarement opéré uniquement quand il est très important avec retentissement sur les structures avoisinantes dont les poumons avec syndrome restrictif. II consiste le plus souvent en une intervention de Nuss avec mise en place d'une barre métallique en arrière du sternum et des côtes pour avancer le thorax. La barre reste en place pendant 3 ans avant d'être retirée. L'intervention est réalisée chez les patients jeunes, avant la puberté et idéalement avant 20 ans pour un meilleur résultat.

Les pieds plats peuvent être corrigés par le port de semelles orthopédiques. Une intervention chirurgicale peut être proposée en cas de forme sévère, jamais avant 8 ans.

La scoliose modérée, inférieure à $20^{\circ}$ est prise en charge par la rééducation. En cas de retentissement plus important, le port d'un corset est proposé. L'indication opératoire ne se pose qu'en cas de scoliose très évoluée, supérieure à $50^{\circ}$, en fin de croissance.

La déminéralisation osseuse est fréquemment observée au cours du syndrome de Marfan. Une ostéodensitométrie est conseillée en cas de présence de facteurs de risque associés tel l'âge (ménopause...) ou de fractures. Des apports optimaux en calcium et vitamine D sont conseillés chez l'adulte et l'enfant.

\subsection{Ophtalmologie (ectopie du cristallin)}

Grâce au suivi mis en route (examen ophtalmologique une fois par an), la survenue d'une cécité est devenue exceptionnelle. L'ablation du cristallin avec pose d'implant est décidée en cas de luxation importante avec retentissement fonctionnel important. La chirurgie de la myopie est contre-indiquée. 


\subsection{Atteinte pulmonaire}

En cas de pneumothorax, le traitement dépend de la gravité des symptômes. En cas de forme modérée, il peut régresser sans traitement. Quand il est plus important, le recours à une exsufflation ou un drainage est possible. Un traitement chirurgical est proposé si le drainage est insuffisant ou en cas de récidive par talcage ou résection de l'un des feuillets de la plèvre. L'arrêt du tabac, facteur favorisant, est fortement conseillé.

\subsection{Croissance de l'enfant}

Pour les enfants avec grande taille, dont la taille prédictive adulte est supérieure ou égale à $1,85 \mathrm{~m}$ chez la fille et à $2 \mathrm{~m}$ chez le garçon, un traitement permettant de limiter la taille finale adulte peut être proposé si l'enfant et sa famille sont demandeurs (somatuline, antihormone de croissance, en injection sous-cutanée tous les 15 jours). Le traitement est mis en route en début de puberté.

Liens d'intérêt : les auteurs ne déclarent aucun lien d'intérêt en rapport avec le sujet. 
Références

1. ORPHANET. Le syndrome de Marfan. https://www.orpha.net/data/patho/Pub/fr/MarfanFRfrPub109.pdf

2. Vanem TT, Geiran OR, Krohg-Sørensen K, Røe C, Paus B, Rand-Hendriksen S. Survival, causes of death, and cardiovascular events in patients with Marfan syndrome. Mol Genet Genomic Med. 2018 doi: 10.1002/mgg3.489.

3. Benoist G, Tubach F, Roy C, et al. Skeletal evolution in Marfan syndrome: growth curves from a French national cohort. Pediatr Res 2018 ; 83 (1-1) : 71-77

4. De Maio F, Fichera A, De Luna V, Mancini F, Caterini R. Orthopaedic Aspects of Marfan Syndrome: The Experience of a Referral Center for Diagnosis of Rare Diseases. Adv Orthop 2016 ;2016: 8275391. 5. Cañadas V, Vilacosta I, Bruna I, Fuster V. Marfan syndrome. Part 2: treatment and management of patients. Nat Rev Cardiol $2010 ; 7: 266-76$.

6. Ledoux M, Beauchet A, Fermanian C, Boileau C, Jondeau G, Saiag P. A case-control study of cutaneous signs in adult patients with Marfan disease : diagnostic value of striae. J Am Acad Dermatol $2011 ; 64: 290-295$

7. Neuville M, Jondeau G, Crestani B, Taillé C. Manifestations respiratoires du syndrome de Marfan. Rev Mal Resp 2015 ; 32 : 173-81.

8. Verstraeten A, Alaerts M, Van Laer L, Loeys B. Marfan Syndrome and Related Disorders: 25 Years of Gene Discovery. Hum Mutat. 2016 ;37:524-31.

9. Jondeau G, Boileau C. Genetics of thoracic aortic aneurysms. Curr Atheroscler Rep. $2012 ; 14$ :21926.

10. Gao LG, Luo F, Hui RT, Zhou XL. Recent molecular biological progress in Marfan syndrome and Marfan-associated disorders. Ageing Res Rev. 2010 ; 9 :363-8. 
11. Aubart M, Gobert D, Aubart-Cohen F et al. Early-onset osteoarthritis, Charcot-Marie-Tooth like neuropathy, autoimmune features, multiarterial aneurysms and dissections: an unrecognized and life-threatening condition. PLoS One 2014 ; 9 : e96387.

12. Stheneur C, Tubach F, Jouneaux M, et al. Study of phenotype evolution during childhood in Marfan syndrome to improve clinical recognition. Genet Med $2014 ; 16: 246-250$

13. von Kodolitsch $\mathrm{Y}$, Rybczynski $\mathrm{M}$, Vogler $\mathrm{M}$, et al. The role of the multidisciplinary health care team in the management of patients with Marfan syndrome. J Multidiscip Healthc 2016 ;9: 587-614. 14. Wanga S, Silversides C, Dore A, de Waard V, Mulder B. Pregnancy and Thoracic Aortic Disease: Managing the Risks. Can J Cardiol. 2016 ;32:78-85.

15. Kim SY, Wolfe DS, Taub CC. Cardiovascular outcomes of pregnancy in Marfan's syndrome patients: A literature review. Congenit Heart Dis. 2018 ; 13:203-209.

16. Rao P, Isselbacher EM. Preconception Counseling for Patients With Thoracic Aortic Aneurysms. Curr Treat Options Cardiovasc Med. 2018 ;20:50.

17. Malfait F, Francomano C, Byers P, et al. The 2017 international classification of the EhlersDanlos syndromes. Am J Med Genet C Semin Med Genet 2017 ;175 : 8-26.

18. De Paepe A, Devereux RB, Dietz HC et al. Revised diagnostic criteria for the Marfan syndrome. Am J Med Genet 1996 ; 62: 417-26.

19. Loeys BL, Dietz HC, Braverman AC et al. The revised Ghent nosology for the Marfan syndrome. J Med Genet 2010 ; 47 : 476-85.

20. von Kodolitsch Y, De Backer J, Schüler H, et al. Perspectives on the revised Ghent criteria for the diagnosis of Marfan syndrome. Appl Clin Genet. 2015 ;8: 137-55.

21. Meester JAN, Verstraeten A, Schepers D, et al. Differences in manifestations of Marfan syndrome, Ehlers-Danlos syndrome, and Loeys-Dietz syndrome. Ann Cardiothorac Surg 2017 ;6:582-594.

22. Maron BJ, Chaitman BR, Ackerman MJ, et al ; Working Groups of the American Heart Association Committee on Exercise, Cardiac Rehabilitation, and Prevention ; Councils on Clinical Cardiology and 
Cardiovascular Disease in the Young. Recommendations for physical activity and recreational sports participation for young patients with genetic cardiovascular diseases. Circulation 2004 ;109:2807-16. 23. Milleron O, Arnoult F, Ropers J et al. Marfan sartan: a randomized, double-blind, placebocontrolled trial. Eur Heart J $2015 ; 36$ : 2160-6.

24. Jondeau G. Recommandations sur la prise en charge médicamenteuse des atteintes aortiques du syndrome de Marfan. Arch Mal Coeur Vaiss 2006 ; 99: 540-546

25. Gaudino M, Girardi LN, Rahouma M, et al. Editor's Choice - Aortic Re-operation After Replacement of the Proximal Aorta: A Systematic Review and Meta-Analysis. Eur J Vasc Endovasc Surg 2018 ;56: 515-523. 


\section{Légendes des figures}

Figure 1. Arachnodactylie : signe dit du pouce (Centre de Référence pour le syndrome de Marfan et apparentés, hôpital Bichat, Paris)

Figure 2. Pectus carinatum (Centre de Référence pour le syndrome de Marfan et apparentés, hôpital Bichat, Paris)

Figure 3. Arachnodactylie. Signes du poignet. Le pouce dépasse en refermant les doigts longs autour de lui ; le signe du poignet est positif quand le pouce chevauche l'index en enserrant le poignet.

(Centre de Référence pour le syndrome de Marfan et apparentés, hôpital Bichat, Paris)

Figure 4. Vergetures dans la région de l'épaule caractéristique du syndrome de Marfan (Centre de Référence pour le syndrome de Marfan et apparentés, hôpital Bichat, Paris)

Figure 5. Pied plat sévère particulier au contexte d'hyperlaxité ligamentaire (Centre de Référence pour le syndrome de Marfan et apparentés, hôpital Bichat, Paris) 


\title{
Encadré 1. Adresses utiles et contacts
}

\author{
Hôpital Bichat (AP-HP) \\ Pr Guillaume JONDEAU, coordinateur \\ Tél. : 0140256811 / Fax : 0140256732 \\ consultation.marfan@bch.aphp.fr \\ www.marfan.fr
}

Centre de Référence pour le syndrome de Marfan et apparentés

PNDS (Protocole National de Diagnostic et de Soins Syndrome de Marfan et apparentés ; www.has-sante.fr

Association MARFANS : www.vivremarfan.org

Orphanet - https://www.orpha.net/data/patho/Pub/fr/Marfan-FRfrPub109.pdf

Réseaux nationaux et européens

Réseaux coordonnés par le Prof Guillaume Jondeau, CRMR Marfan :

Filière nationale Maladies vasculaires rares avec atteinte multi systémique :

www.FAVAmulti.fr

Réseau européen de référence (ERN) Maladies Vasculaires Rares : www.VASCERN.eu 
Tableau 1 : mutations génétiques en dehors de FBN1 avec caractéristiques cliniques particulières

\begin{tabular}{|c|c|c|}
\hline $\begin{array}{c}\text { TGFBR1 (Syndrome de Loeys- } \\
\text { Dietz) }\end{array}$ & SMAD 3 & АСТА 2 \\
\hline $\begin{array}{l}\text { Luette bifide } \\
\text { Hypertélorisme } \\
\text { Signes musculo- } \\
\text { squelettiques moins } \\
\text { importants }\end{array}$ & $\begin{array}{l}\text { Arthrose précoce }<40 \text { ans } \\
\text { avec lésions radiologiques } \\
\text { de type rhumatisme } \\
\text { microcristallin }\end{array}$ & \\
\hline $\begin{array}{l}\text { Peau fine, retard à la } \\
\text { cicatrisation }\end{array}$ & $\begin{array}{l}\text { Neuropathie type Charcot- } \\
\text { Marie-Tooth } \\
\text { Asthme } \\
\text { Conjonctivite allergique } \\
\text { Troubles veineux } \\
\text { Anomalies auto-immunes } \\
\text { (Syndrome de Sjögren } \\
\text { primitif, thyroïdite } \\
\text { d'Hashimoto, FAN+ ...) }\end{array}$ & Livedo racemosa \\
\hline $\begin{array}{l}\text { Tortuosité artérielle } \\
\text { généralisée, principalement } \\
\text { tête et cou } \\
\text { Risque de } \\
\text { dissection/rupture à un } \\
\text { diamètre + faible et à un } \\
\text { âge + précoce } \\
\text { Pas d'ectopie du cristallin, } \\
\text { myopie moins sévère }\end{array}$ & & \\
\hline
\end{tabular}


TABLEAU 2 - Critères diagnostiques du syndrome de Marfan adapté des critères de Gand (Ghent 2010).

\begin{tabular}{|c|c|c|}
\hline \multirow[t]{2}{*}{ Critères } & \multicolumn{2}{|c|}{ Signes } \\
\hline & Majeurs & Mineurs \\
\hline $\begin{array}{l}\text { Squelettiques: } \\
\text { Au moins } 4 \text { signes majeurs } \\
\text { (pour une atteinte squelettique majeure), } \\
\text { ou } 2 \text { signes majeurs }+1 \text { signe mineur ou } 1 \\
\text { signe majeur }+2 \text { signes mineurs (pour une } \\
\text { atteinte mineure) }\end{array}$ & $\begin{array}{l}\text { Pectus carinatum } \\
\text { Pectus excavatum nécessitant la chirurgie } \\
\text { Rapport segment supérieur sur segment } \\
\text { inférieur bas ou envergure (membres } \\
\text { supérieurs écartés) sur taille supérieure à } 1,05 \\
\text { Signe du poignet ou du pouce } \\
\text { Scoliose supérieure à } 20^{\circ} \text { ou spondylolisthésis } \\
\text { Extension maximale des coudes inférieure à } \\
170^{\circ} \text { (flessum) } \\
\text { Pieds plats } \\
\text { Protrusion acétabulaire }\end{array}$ & $\begin{array}{l}\text { Pectus excavatum modéré } \\
\text { Hyperlaxité ligamentaire } \\
\text { Palais ogival avec chevauchement des dents } \\
\text { Faciès caractéristique }\end{array}$ \\
\hline $\begin{array}{l}\text { Oculaire : } \\
1 \text { signe majeur ou } 2 \text { mineurs }\end{array}$ & Ectopie du cristallin & $\begin{array}{l}\text { Cornée plate } \\
\text { Globe oculaire allongé } \\
\text { Iris hypoplasique ou hypoplasie du muscle } \\
\text { ciliaire }\end{array}$ \\
\hline $\begin{array}{l}\text { Cardiovasculaire : } \\
\text { Au moins } 1 \text { signe }\end{array}$ & $\begin{array}{l}\text { Dilatation de l'aorte ascendante intéressant les } \\
\text { sinus de Valsalva } \\
\text { Dissection aortique }\end{array}$ & $\begin{array}{l}\text { Insuffisance aortique } \\
\text { Prolapsus valvulaire mitral avec ou sans fuite } \\
\text { Dilatation de l'artère pulmonaire avant l'âge } \\
\text { de } 40 \text { ans } \\
\text { Calcifications de l'anneau mitral avant l'âge de } \\
40 \text { ans } \\
\text { Anévrisme ou dissection de l'aorte } \\
\text { abdominale avant l'âge de } 50 \text { ans }\end{array}$ \\
\hline $\begin{array}{l}\text { Pulmonaire : } \\
\text { Au moins } 1 \text { signe Mineur }\end{array}$ & & $\begin{array}{l}\text { Pneumothorax spontané } \\
\text { Bulle apicale }\end{array}$ \\
\hline $\begin{array}{l}\text { Cutanée : } \\
\text { Au moins } 1 \text { signe }\end{array}$ & & $\begin{array}{l}\text { Vergetures (à l'exclusion de grossesse, perte } \\
\text { de poids) } \\
\text { Hernies récidivantes ou chirurgicales }\end{array}$ \\
\hline
\end{tabular}




\begin{tabular}{|l|l|l|}
\hline $\begin{array}{l}\text { Neurologique : } \\
\text { Au moins } 1 \text { signe }\end{array}$ & Ectasie de la dure-mère lombo-sacrée & $\begin{array}{l}\text { Méningocèle et/ou élargissement du fourreau } \\
\text { dural } \\
\text { Kystes radiculaires étagés } \\
\text { Amincissement des pédicules } \\
\text { Scalloping } \\
\text { Disparition de la graisse périradiculaire }\end{array}$ \\
\hline $\begin{array}{l}\text { Histoire familiale/génétique : } \\
1 \text { signe majeur }\end{array}$ & $\begin{array}{l}\text { Un parent direct ayant les critères } \\
\text { diagnostiques } \\
\text { Mutation de FBN1 déjà connue pour provoquer } \\
\text { un syndrome de Marfan ou mutation } \\
\text { TGFbétaR1 ou R2, présence d'un marqueur } \\
\text { génétique, proche du gène de la fibrilline de } \\
\text { type } 1 \text {, se transmettant avec la maladie dans la } \\
\text { famille. }\end{array}$ \\
\hline $\begin{array}{l}\text { Interprétation des critères diagnostiques: } \\
\text { Le diagnostic de Marfan est posé }\end{array}$ & $\begin{array}{l}\text { En cas d'histoire familiale devant : } 1 \text { critère majeur }+1 \text { autre système atteint } \\
\text { En l'absence d'histoire familiale devant : } 1 \text { critère majeur dans } 2 \text { systèmes }+1 \text { autre système } \\
\text { atteint ou mutation FBN1 } 1 \text { critère majeur dans } 1 \text { système }+1 \text { autre système atteint }\end{array}$ \\
\hline
\end{tabular}




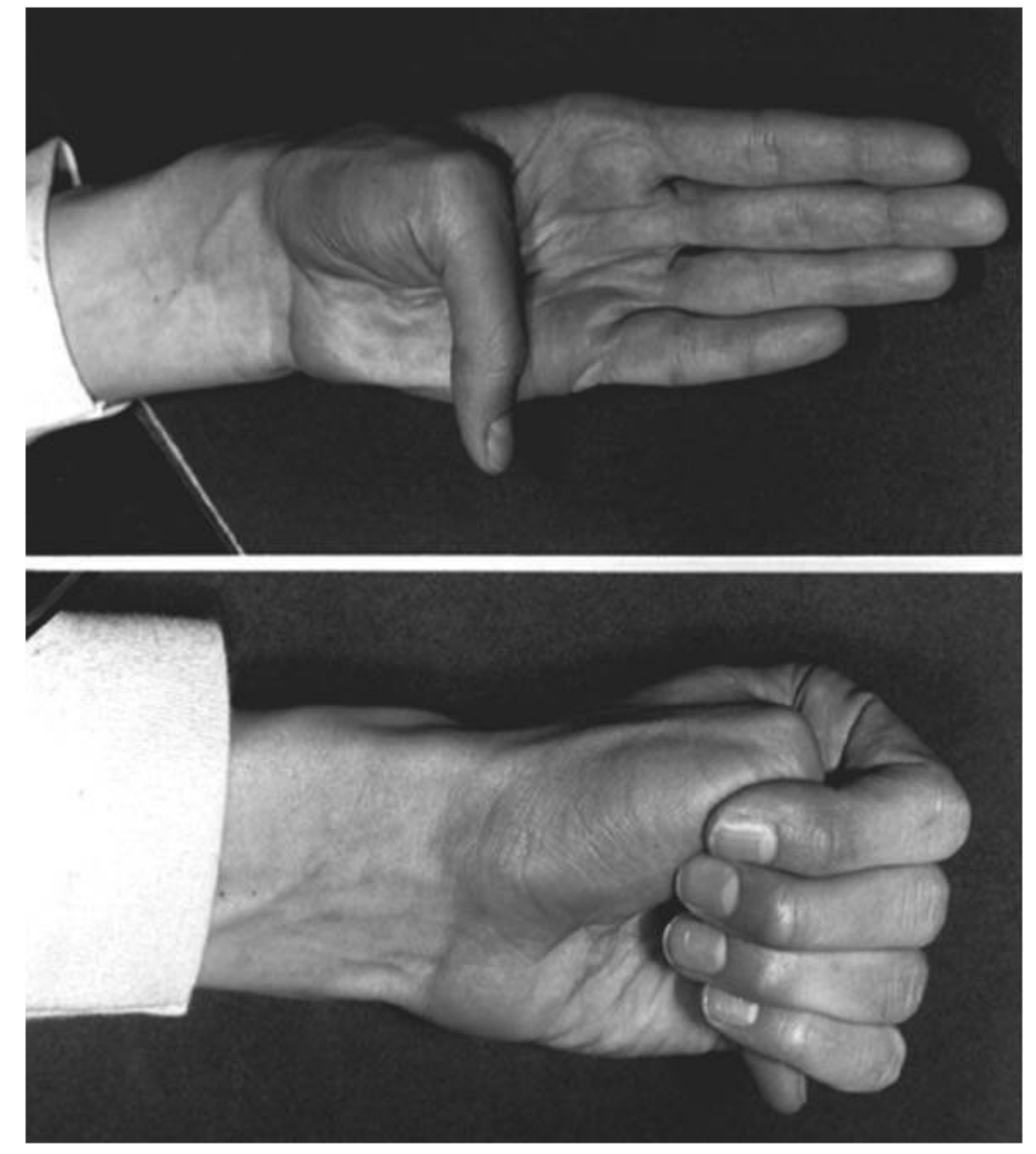




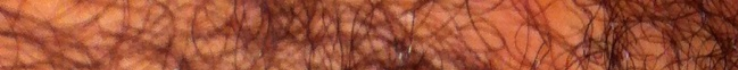
$52,-0 x=1$

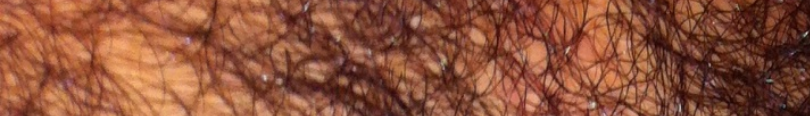

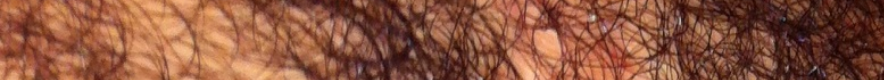
$x+2 x+100$

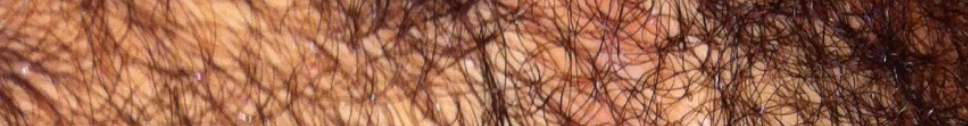

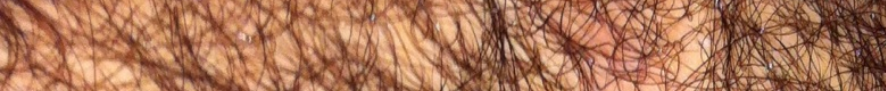

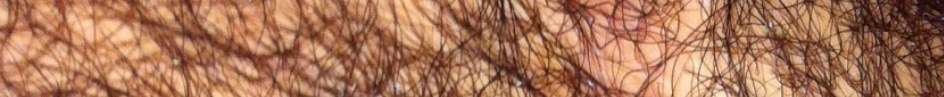

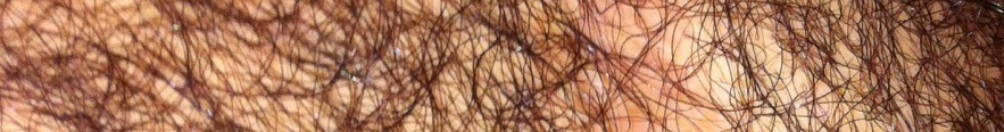

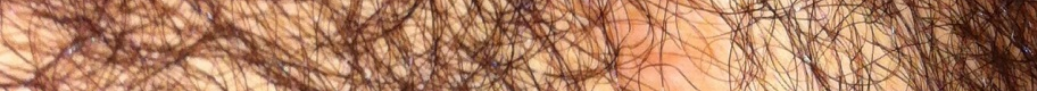

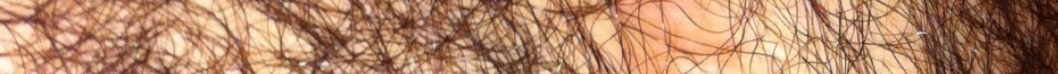
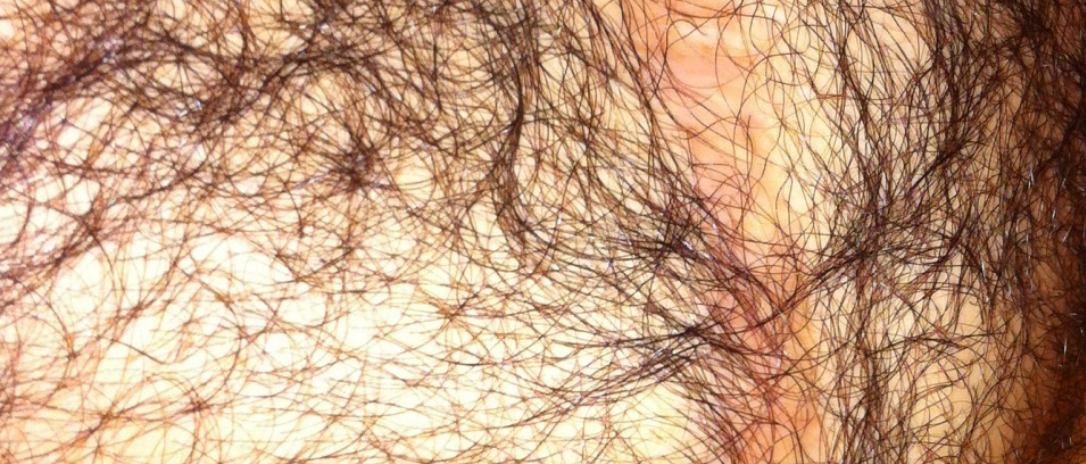

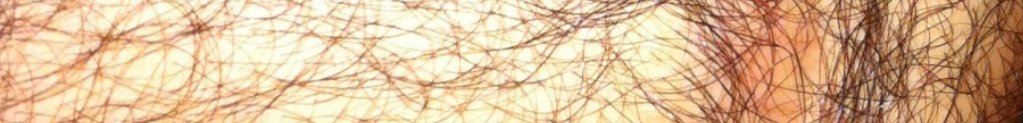
2. $2+2 \times 1)$ 


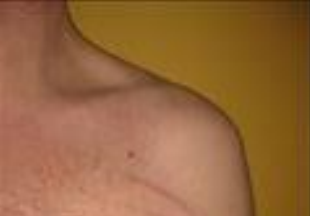

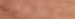

$x+\frac{1}{2}$

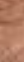

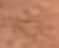

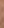

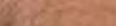

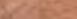

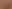

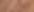

$\sqrt{x+2}$

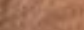

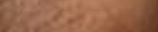

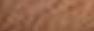



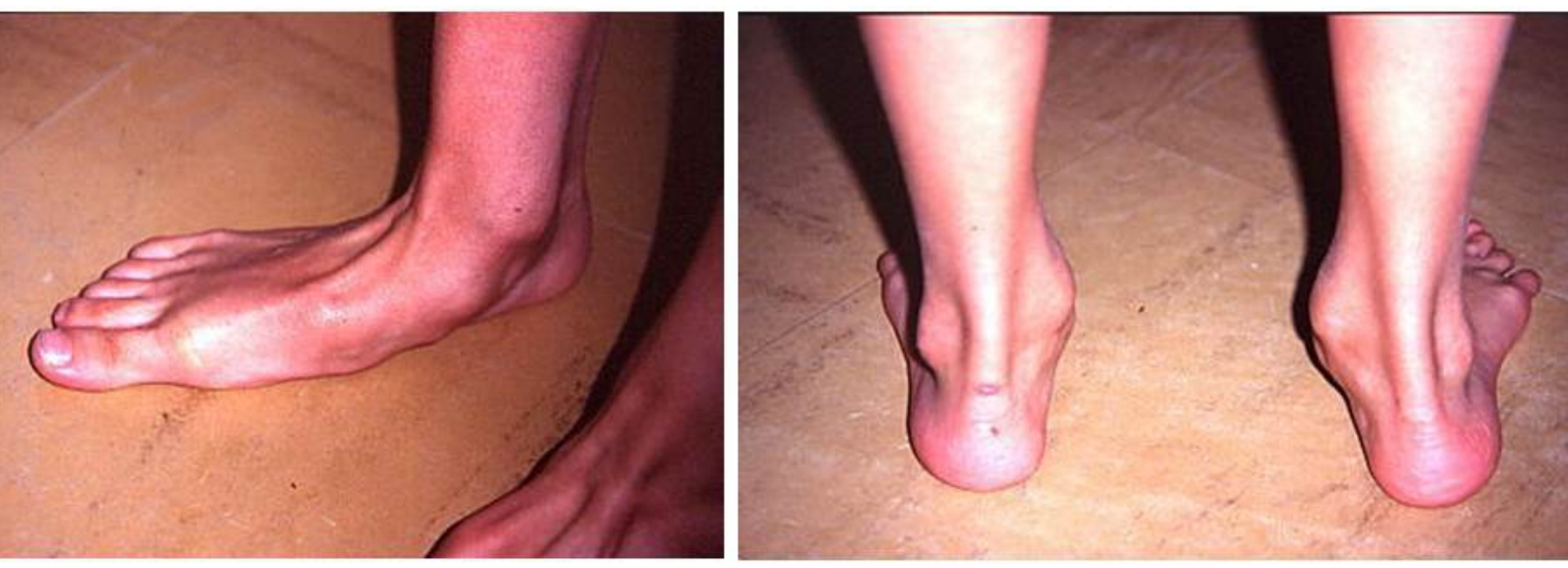